\title{
Coronary artery calcium scanning in symptomatic patients: Ready for use as a gatekeeper for further testing?
}

\author{
Alan Rozanski, MD, ${ }^{\mathrm{a}, \mathrm{b}}$ and Daniel S. Berman, $\mathrm{MD}^{\mathrm{c}}$ \\ a Division of Cardiology, Mount Sinai St. Lukes Hospital, Mount Sinai Heart, New York, NY \\ b Icahn School of Medicine at Mount Sinai, New York, NY \\ c Departments of Imaging and Medicine, Cedars-Sinai Medical Center and the Cedars-Sinai \\ Heart Institute, Los Angeles, CA
}

Received Jan 8, 2017; accepted Jan 9, 2017

doi: 10.1007/s12350-017-0794-2

Coronary artery calcium (CAC) scanning was first introduced into medicine as a screening test for coronary artery disease (CAD). As an example of "first impressions really count," this reputation has really stuck. To date, the vast majority of epidemiological work concerning CAC scanning has centered upon its use as a screening tool for CAD. Now, however, there is growing interest in assessing other potential uses of CAC scanning including its potential use in symptomatic patients as a gatekeeper for further noninvasive testing. In this issue of the Journal, two sets of authors take a protagonist and antagonist view toward the question of whether CAC scanning can serve as such a gatekeeper.

Engbers et al take a protagonist position. ${ }^{1}$ They cite numerous studies concerning the application of CAC in nearly 15,000 symptomatic patients with respect to detection of obstructive coronary stenosis as defined by coronary CT angiography (CCTA) or invasive coronary angiography. In these patients, a zero CAC score was rarely associated with obstructive $\mathrm{CAD}$. They proceed to integrate the CAC score into an argument that its use in patients with chest pain depends on the pre-test likelihood of obstructive CAD. They further expand on the type of testing-stress MPI or CCTA - that might be used after CAC testing in patients with CAC scores greater than zero. They suggest the application of CCTA for patients with CAC scores 1-600, the application of MPI for patients with CAC 600-1000, and either stress MPI or direct invasive coronary angiography for patients with

\footnotetext{
Reprint requests: Alan Rozanski, MD, Division of Cardiology, Mount Sinai St. Lukes Hospital, Mount Sinai Heart, 1111 Amsterdam Avenue, New York, NY 10025, USA; arozanski@chpnet.org J Nucl Cardiol 2017;24:835-8. $1071-3581 / \$ 34.00$

Copyright (c) 2017 American Society of Nuclear Cardiology.
}

CAC $>1000$. Ruckel and Gulati take an antagonist view and offer several reasons to support their point of view. ${ }^{2}$ First, they cite inconsistent data regarding the ability of a zero CAC score to exclude obstructive CAD. Second, they make the point that anatomic lesions alone are not always responsible for ischemia, especially in women. Thus, they argue that reliance on CAC scanning, an anatomic test, could result in a missed identification of either obstructive $\mathrm{CAD}$ or ischemia were it to serve as a gatekeeper test. Third, they note that the impact of using CAC scanning compared to functional testing upon clinical outcomes for the work-up of patients presenting with chest pain is untested and thus not known.

There are some important points of common ground among the authors. Both groups heavily focus on the issue of whether a zero CAC score can exclude obstructive CAD in their debate. They both cite evidence that the ability of a zero CAC score to exclude obstructive CAD is highly dependent on the pre-test likelihood of CAD. That is, among populations in whom the prevalence of obstructive CAD is low, a zero CAC score is effective in reducing the likelihood of obstructive CAD to very low levels. By contrast, when the prevalence of CAD is much higher, such as in patients being referred for cardiac catheterization, a zero CAC score is less effective in excluding the presence of obstructive CAD. Thus, we can sharpen their debate as follows: Is CAC scanning an effective gatekeeper for further testing among patients with a low-intermediate likelihood of obstructive CAD?

\section{THE INTRINSIC EFFICACY OF CORONARY ARTERY CALCIUM SCANNING}

CAC scanning has intrinsic properties that make it potentially useful for applications beyond its 
conventional use in screening populations. CAC is a highly specific marker for the presence of underlying atherosclerosis, representing the integration of the effects of all risk factors over a lifetime in an individual patient. CAC scanning is also a highly sensitive method for detecting atherosclerosis, although this sensitivity is dependent on several factors. For example, sensitivity is low in young patients who tend to have more noncalcified plaques and, as noted above, its absence does not completely exclude the presence of obstructive $\mathrm{CAD}$ in patients with a higher pre-test likelihood of CAD. However, in general, for populations with a low-tointermediate likelihood of disease, the CAC scan is a highly sensitive detector of atherosclerosis. Importantly, even a CAC score of 1 establishes the presence of atherosclerosis, and a CAC score of only 1-10 is already sufficient to double the risk for cardiac events compared to a CAC score of zero. ${ }^{3,4}$

Another great strength of the CAC scan is its ability to reflect the total burden of coronary atherosclerosis. ${ }^{5}$ Cardiac risk increases in direct proportion to increasing degrees of CAC abnormality. ${ }^{6,7}$ Moreover, since CAC actually detects and measures atherosclerosis, whereas CAD risk factors merely attempt to predict the presence of disease, CAC scanning has an intrinsically greater capacity than CAD risk factors to predict cardiac events, and adds incremental information to the assessment of risk when CAD risk factors are first considered. The CAC scan is now widely accepted as having high efficacy and as being a highly effective tool for detecting subclinical atherosclerosis and guiding preventive therapy, including by the authors of the present debate.

\section{THE BASIS FOR CONSIDERING CAC SCANNING AS A GATEKEEPER FOR FURTHER TESTING}

Three factors suggest a potential utility for CAC scanning as a gatekeeper for more advanced testing. First, greater selection is needed in determining which patients should be referred for advanced imaging tests. Over the last few decades, there has been a marked temporal decline in the frequency of inducible myocardial ischemia in patients referred for myocardial perfusion imaging. We observed in the Cedars-Sinai database over a 19-year period a progressive fall in the observed frequency of inducible ischemia among patients referred for SPECT-MPI, from a prevalence of $30 \%$ in 1991 to just 5\% in 2009 (Figure 1), ${ }^{8}$ including in each category of pre-test CAD likelihood. Further, the observed prevalence of obstructive CAD has also decreased in each category of CAD likelihood as assessed by the original Diamond-Forrester criteria that are still used in clinical practice today. In the large CONFIRM registry of patients undergoing coronary

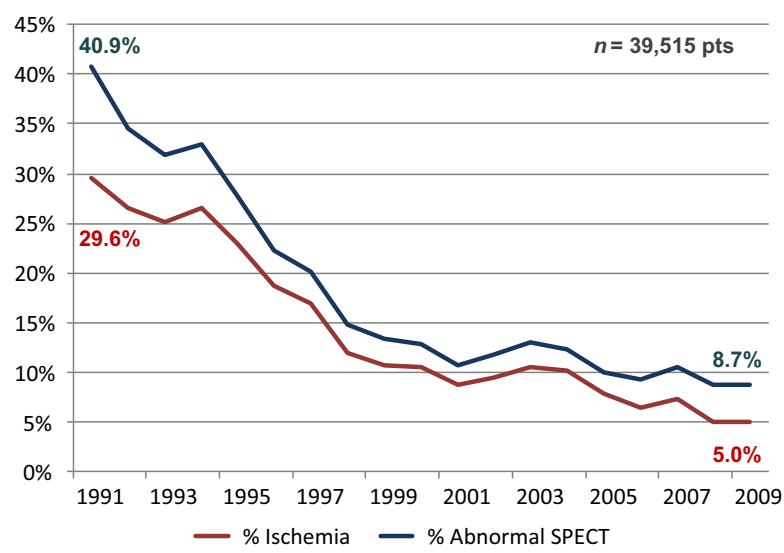

Figure 1. Progressive temporal decrease in the frequency of an abnormal SPECT myocardial perfusion study (blue) and in the frequency of inducible myocardial ischemia among patients referred for radionuclide stress testing because of suspected coronary artery disease between 1991 and 2009. From Rozanski et al. ${ }^{8}$.

CTA, Chang et al demonstrated a markedly lower prevalence of obstructive $\mathrm{CAD}$, as measured by coronary CTA, among patients stratified by each age, gender, and chest pain category compared to the DiamondForrester algorithm (D-F 1979). ${ }^{9}$ In fact, only men over age 60 with typical angina had an observed prevalence of $\geq 50 \%$ stenosis that exceeded $50 \%$ in this registry. Similarly, in the PROMISE trial, while the pre-test likelihood of CAD was $53 \%$ by the Diamond-Forrester criteria in the coronary CTA arm, $\geq 50 \%$ stenosis was found in only $10.3 \%$ of the patients undergoing CTA. ${ }^{10}$ Thus, most patients being referred for noninvasive imaging at the present time fit into the category of either a low or low-intermediate likelihood of obstructive CAD.

Second, while the frequency of obstructive CAD and overt ischemia has now declined, current patients referred for cardiac testing still have a burden of atherosclerosis, which is often substantial in magnitude even among those without inducible ischemia. For example, in a meta-analysis of 20 studies that compared the results of CAC scanning to those of SPECT-MPI, Bavishi et al observed among patients with a normal SPECT-MPI study that $72 \%$ of patients had CAC $>0$ and $25 \%$ had CAC scores $>400 .{ }^{11}$ As an aside, in this population, the knowledge of the CAC score $>0$ can allow institution of preventive therapies (e.g., statins, aspirin), potentially avoiding one of the major limitations of ischemia testing - the failure to identify patients with subclinical atherosclerosis.

This observation becomes important when considering a third point: the presence of a proportional relationship between the magnitude of CAC abnormality 
and the likelihood of inducible myocardial ischemia. ${ }^{11-13}$ Generally, the absence of CAC or very low CAC scores tends to lower patients' likelihood of inducible myocardial ischemia, whereas the frequency of ischemia tends to become progressively substantial with high CAC scores $(>400)$. Thus, consideration of the CAC score might be used to help decide the need for subsequent cardiac stress testing.

However, because of the long-term focus on using CAC scanning as a screening test, there has to date been little focus on how CAC scanning might specifically be applied as a triage tool for further testing, whether it be the use of exercise ECG, coronary CT angiography, or cardiac stress imaging (echo, SPECT-MPI, or PETMPI). For instance, there is presently a large variance in reported frequencies of ischemia according to CAC abnormality that needs to be further explored. ${ }^{11}$ In addition, there has been a relative paucity of study that examines how clinical factors modulate the relationship between CAC scores and ischemia, such as diabetic status and chest pain. ${ }^{14,15}$ Most importantly, the presumed effectiveness of CAC testing is likely to be markedly influenced by the population to which the test is being applied. However, because of the now very low cost of this combined testing and low radiation exposure of CAC scanning, this approach deserves more active consideration as a means of minimizing unnecessary diagnostic testing. While prospective study will be required, some reasonable suggestions can be made as to when the use of CAC scanning might prove effective as a gatekeeper to further testing:

\section{For Asymptomatic Patients with Risk Factors (Not Part of the Current Debate)}

In this group, the ACC/AHA multimodality guidelines currently consider exercise testing as appropriate and stress imaging or CCTA as "maybe" appropriate. ${ }^{16}$ However, extensive data with CAC scanning, the least expensive of these approaches, show that a very large proportion of patients with three or more risk factors have zero CAC scores and a very low risk of cardiac events. ${ }^{17}$ CAC scanning, possibly in conjunction with treadmill testing, could thus be effective in these patients in ruling out the need for advanced imaging procedures.

\section{For Stable Symptomatic Patients with a Low-Intermediate Likelihood of Obstructive CAD}

In symptomatic patients with low-to-intermediate pre-test likelihood of obstructive CAD which as noted applies to the vast majority of patients currently being referred for advanced imaging procedures, a CAC score of zero would generally signify no need for further testing.

However, once CAC is present, options for testing become more varied. Among a patient with bonafide low risk (e.g., nonanginal chest pain) and a very low CAC score (e.g., 1-50), some might suggest no further testing is required, while others might prefer to add an exercise ECG to further confirm low risk. Then as CAC scores rise to low-intermediate values $(\sim 100)$ in the same presenting patient, the addition of exercise ECG might gain proportionately in utility. If treadmill testing is then normal and exercise duration adequate, further testing would generally not be needed, but would be appropriate for those with stress-induced symptoms, an ischemic ECG response, or for those with low exercise capacity. ${ }^{18}$ Alternatively, coronary CTA may also become a favored test in this group, pending further study.

As the CAC scores increase to the higher end of this intermediate range, the case for advanced testing with either CTA or stress testing with either MPI or echo may be preferred due to the increasing clinical risk of such patients. Finally, higher CAC scores $(>600$ or $>1000)$ may define a group in which MPI becomes favored over CTA, as the proportion of patients with nondiagnostic CTA studies is increased in this group.

This proposed guideline is deliberately tentative due to the need for prospective studies that would test this guideline for cost-effectiveness. Other guidelines might also be proposed and tested. As noted, the aforementioned algorithm of Embers et al that proposes that patients with CAC scores from 1-600 should be considered for coronary CT angiography represents one such alternative paradigm that could also be tested by prospective study, ${ }^{1}$ but its proposal to use CTA in the presence of low CAC scores raises the possibility of leading to more testing and intervention than necessary. The possibility of adding a predictor of fractional flow reserve from the coronary CTA (FFRct) as a means of reducing subsequent unnecessary invasive coronary angiography has recently been described in this regard. ${ }^{19}$

\section{For Patients with Higher CAD Likelihood}

In patients, whose clinical presentation suggests a high-intermediate pre-test likelihood of CAD, such as patients in an older age group with recent onset or worsening typical angina, CAC scanning alone would not be recommended for initial triage, as it would neither exclude obstructive CAD nor accurately predict its presence. Rather, in such patients, stress testing or noninvasive coronary CT angiography would be appropriate as a first line strategy, particularly in the elderly, as excessive CAC increases the frequency of nondiagnostic 
coronary CTA studies. Nonetheless, CAC scanning can be highly useful in conjunction with SPECT or PET-MPI in this population. If a normal MPI study is found in a patients with very high CAC scores (e.g., over $>1000$ ), the possibility of residual high risk may exist. ${ }^{20}$

These proposals require "real world" testing to determine their validity in the form of comparative effectiveness research that would compare such proposed paradigms vs the usual standard of care for the work-up patients presenting with chest pain.

\section{CONCLUSION}

In the debate between the protagonists and antagonists to use CAC scanning as a gatekeeper for further testing among chest pain patients with a low-intermediate likelihood of CAD, who is right? Essential to knowing which pathway to use in selection of a testing strategy is an accurate assessment of the clinical starting point regarding the pre-test likelihood of CAD. Regarding the use of CAC scanning, Engbers et al are right to suggest that there is potential to improve the costeffectiveness of testing by using CAC scanning as a triage for further more advanced testing in such patients. Ruckel and Gulati, however, are right to point out that this supposition requires prospective evaluation to assess its potential impact on patients' clinical outcomes.

\section{Acknowledgments}

Funded, in part, by the Dr. Miriam and Sheldon G. Adelson Medical Research Foundation and the Diane and Guilford Glazer Foundation.

\section{Disclosures}

There is no relationship with industry for this manuscript.

\section{References}

1. Engbers EM. Timmer JR, Ottervanger JP. Coronary artery calcium score as a gatekeeper in the non-invasive evaluation of suspected coronary artery disease in symptomatic patients. Journal of Nuclear Cardiology. 2017. doi:10.1007/s12350-017-0792-4.

2. Ruckel S, Gulati M. Coronary calcium scoring as the gate keeper for stress myocardial perfusion imaging antagonist. Journal of Nuclear Cardiology. 2017. doi:10.1007/s12350-017-0793-3.

3. Budoff MJ, Mcclelland R, Nasir K, et al. Cardiovascular events with absent or minimal coronary calcification: The multi-ethnic study of atherosclerosis (MESA). Am Heart J. 2009;158:554.

4. Blaha M, Budoff MJ, Shaw LJ, et al. Absence of coronary artery calcification and all-cause mortality. JACC Cardiovasc Imaging. 2009;2:692-700.

5. Rumberger JA, Simons DB, Fitzpatrick LA, Sheedy PF, Schwartz RS. Coronary artery calcium area by electron-beam computed tomography and coronary atherosclerotic plaque area: A histopathologic correlative study. Circulation. 1995;92:2157-62.

6. Budoff MJ, Shaw LJ, Liu ST, et al. Long-term prognosis associated with coronary calcification: Observations from a registry of 25,253 patients. J Am Coll Cardiol. 2007;49:1860-70.

7. Detrano R, Guerci AD, Carr JJ, et al. Coronary calcium as a predictor of coronary events in four racial or ethnic groups. The New England Journal of Medicine. 2008;358:1336-45.

8. Rozanski A, Gransar H, Hayes SW, et al. Temporal trends in the frequency of inducible myocardial ischemia during cardiac stress testing: 1991 to 2009. J Am Coll Cardiol. 2013;61:1054-65.

9. Cheng VY, Berman DS, Rozanski A, et al. Performance of the traditional age, sex, and angina typically-based approach for estimating pretest probability of angiographically significant coronary artery disease in patients undergoing coronary computed tomographic angiograpghy: Results from the multinational coronary CT angiography evaluation for clinical outcomes: An international multicenter registry (CONFIRM). Circulation. 2011;124:1-8.

10. Douglas PS, Hoffmann U, Patel MR, et al. Outcomes of anatomical versus functional testing for coronary artery disease. N Engl J Med. 2015;372:1291-300.

11. Bavishi C, Argulian E, Chatterjee S, Rozanski A. Cacs and the frequency of stress-induced myocardial ischemia during MPI: A meta-analysis. JACC Cardiovasc Imaging. 2016;9:580-9.

12. Berman DS, Wong ND, Gransar H, et al. Relationship between stress-induced myocardial ischemia and atherosclerosis measured by coronary calcium tomography. J Am Coll Cardiol. 2004;44:923-30.

13. Chang SM, Nabi F, Xu J, et al. The coronary artery calcium score and stress myocardial perfusion imaging provide independent and complementary prediction of cardiac risk. J Am Coll Cardiol. 2009;54:1872-82.

14. Wong ND, Rozanski A, Gransar H, Dahlbeck J, Miranda-Peats R, Hayes S, Shaw L, Friedman J, Polk D, Berman DS. Metabolic syndrome and diabetes are associated with an increased likelihood of inducible myocardial ischemia among patients with subclinical atherosclerosis. Diabetes Care. 2005;78:1445-50.

15. Rozanski A, Gransar H, Wong ND, et al. Use of coronary calcium scanning for predicting inducible myocardial ischemia: Influence of patients' clinical presentation. J Nucl Cardiol. 2007;14:669-79.

16. Ronan G, Wolk MJ, Bailey SR, et al. ACCF/AHA/ASE/ASNC/ HFSA/HRS/ SCAI/SCCT/SCMR/STS 2013 multimodality appropriate use criteria for the detection and risk assessment of stable ischemic heart disease: A report of the american college of cardiology foundation appropriate use criteria task force, american heart association, american society of echocardiography, american society of nuclear cardiology, heart failure society of America, heart rhythm society, society for cardiovascular angiography and interventions, society of cardiovascular computed tomography, society for cardiovascular magnetic resonance, and society of thoracic surgeons. J Nucl Cardiol. 2014;21:192-220.

17. Nasir K, Rubin J, Blaha MJ, et al. Interplay of coronary artery calcification and traditional risk factors for the prediction of allcause mortality in asymptomatic individuals. Circ Cardiovasc Imaging. 2012;5:467-73.

18. Rozanski A, Cohen RA, Uretsky S. The coronary calcium treadmill test: A new approach to the initial work-up of patients with suspected coronary artery disease. J Nucl Cardiol. 2013;20:719-30.

19. Hecht HS, Narula J, Fearon WF. Fractional flow reserve and coronary computed tomographic angiography. A review and critical analysis. Circ Res. 2016;119:300-16.

20. Yuoness SA, Goha AM, Romsa JG, et al. Very high coronary artery calcium score with normal myocardial perfusion SPECT imaging is associated with a moderate incidence of severe coronary artery disease. Eur J Nucl Med Mol Imaging. 2015;42:1542-50. 